\title{
CONSTRUÇÃO PARTICIPATIVA DE UMA POLÍTICA DE SAÚDE PARA INSTITUTOS FEDERAIS
}

\section{Participatory construction of a health policy for Federal Institute}

Flávia Christiane de Azevedo Machado - Doutora em Saúde Coletiva/Professora Adjunta UFRN. E-mail: flavitamachado@yahoo.com.br

Janmille Valdivino Silva - Mestre em Saúde Coletival Técnico administrativo IFRN. E-mail: janmillevs@yahoo.com.br

Gerliene Maria Silva Araújo de Almeida - Mestre em Saúde Coletiva/Técnico administrativo IFRN. E-mail: gerliene@gmail.com

Ana Cristina Gondim Filgueira - Mestre em Saúde Coletiva/Assistente administrativo IFRN. E-mail: ana.filgueira@ifrn.edu.br

Ideíze de Barros Medeiros - Especialista em Ambiente Organizacional, Saúde e Ergonomia / Técnico Administrativo IFRN. E-mail: ideize.medeiros@ifrn.edu.br

Thiago Antonio Raulino do Nascimento - Mestre em Demografia/Técnico administrativo IFRN. E-mail: thiago.nascimento@ifrn.edu.br

Autor responsável pela correspondência:

Flávia Christiane de Azevedo Machado. E-mail: flavitamachado@yahoo.com.br 


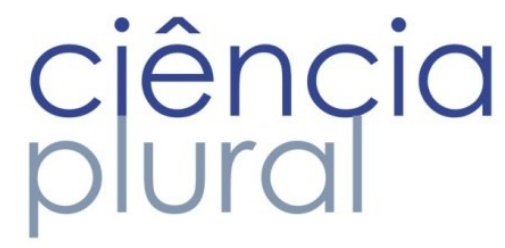

RESUMO

Introdução: No Brasil, quando se mencionam serviços públicos de saúde nos remetemos aos serviços do Sistema Único de Saúde - SUS. Contudo, há instituições públicas, não integrantes do SUS, que disponibilizam serviços de saúde e, assim, fariam jus a uma política de saúde específica. Objetivo: Reportar a estruturação de uma política de saúde para a assistência estudantil do Instituto Federal Educação, Ciência e Tecnologia do Rio Grande do Norte (IFRN) mediante construção de uma matriz de indicadores. Métodos: Estudo de avaliação cujo processo de criação da política foi: análise documental para definir missão e atribuições dos setores de saúde/IFRN; consulta a inquéritos nacionais (SB Brasil, Saúde na Escola, CENSO), relatório socioeconômico aluno/IFRN e análise dos recursos/IFRN (questionário Google forms $®$ ) para 0 diagnóstico situacional; Definição de diretrizes/objetivos/problemas-alvo; Construção de matriz de indicadores; validação da matriz por coordenadores/profissionais de saúde/IFRN via técnica conferência consenso; criação de instrumentos de monitoramento (programação anual de saúde, relatório de execução). Resultados: As diretrizes foram legitimadas, 01 objetivo foi excluído e 01 modificado. Quanto aos indicadores, houve redução de 42 para 37 tidos como pertinentes/válidos/oportunos. Conclusão: Para viabilização da política são necessárias estratégias para institucionalização da educação em saúde, da pesquisa científica, dos processos de avaliação/planejamento/monitoramento, normatização do funcionamento dos serviços e 0 desenvolvimento de ações intersetoriais.

Palavras-chave: Política de Saúde, Gestão em Saúde, Avaliação de Serviços de Saúde, Instituições de ensino, Atenção à Saúde.

\section{ABSTRACT}

Introduction: In Brazil, when referring to public health services we usually refer to the services of the Unified Health System - SUS. However, there are public institutions, not members of the SUS, that provide health services and should hav a specific health policy. Objective: To report the structuring of a health policy for the student assistance of the Federal Institute of Education, Science and Technology of Rio Grande do Norte (IFRN) through the construction of a matrix of indicators. Method: Evaluation study whose policy creation process was: document analysis to define IFRN health sector's mission and responsibilities; consultation on national surveys (OH Brazil, School Health; CENSUS), to IFRN student's socioeconomic report and analysis of IFRN's resources (Google forms $®$ questionnaire) for the situational diagnosis; definition of guidelines/objectives/target problems; construction of an indicator's matrix; validation of the matrix by IFRN's coordinators/health professionals by technical consensus conference; creation of monitoring instruments (annual health programming, implementation report). Results: The guidelines were legitimized, 01 objective was excluded and 01 was modified. As for the indicators, there was a reduction of 42 to 37 taken as relevant/valid/timely. Conclusion: Implementing the policy requires necessary strategies to institutionalize health education process, scientific research, assessment/planning/monitoring process, standardization of service's operation and the development of intersectoral actions.

Keywords: Health Policy, Health Management, Health Services Evaluation, Schools, Health Care. 


\section{Introdução}

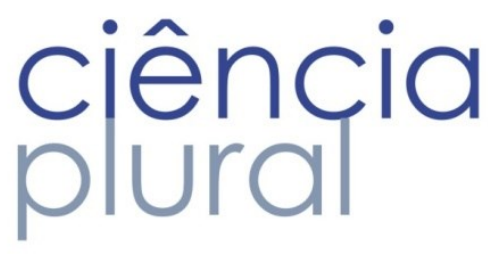

Uma política pública deve considerar a quem se destinam os resultados ou benefícios e o seu processo de elaboração deve ser submetido ao debate público. Elaborar uma política pública significa dizer quem decide o quê, quando, com que consequências e para quem. Os elementos fundamentais na construção concreta de uma política são: diagnóstico da realidade; objetivos pretendidos; e estratégia a ser adotada 1.

As políticas públicas de saúde são políticas sociais desempenhadas de acordo com normas legalmente construídas tendo por objetivo maior a qualidade de vida da população. Essas normas marcos reguladores (institucionais) - representam sustentáculos compostos por referenciais valorativos, políticos, organizacionais e econômicos que permeiam e sustentam a política de saúde e a interligam ao sistema de proteção social com um todo. Assim, o fundamental na construção de uma política é planejar e avaliar estrategicamente para se ter um ganho na capacidade de gestão.

A capacidade de gestão é o potencial para operar os recursos existentes, adquirir novos recursos e mobilizar os recursos oriundos da posição que o gestor ocupa. Assim, o intuito maior é controlar, negociar e articular, com outros atores no processo de tomada de decisão, ações para alcançar os objetivos e metas planejados. Portanto, a capacidade de gestão refere-se ao exercício do poder e à sua legitimidade².

No Brasil, quando se mencionam serviços públicos de saúde nos remetemos aos serviços do Sistema Único de Saúde - SUS. Contudo, há instituições públicas, não integrantes do SUS, que disponibilizam serviços de saúde e, assim, fariam jus a uma política de saúde específica. Dentre essas instituições, identificam-se os Institutos Federais de Educação, Ciência e Tecnologia - IF. Os IF são instituições de educação superior, básica e profissional especializadas na oferta de educação profissional e tecnológica nas diferentes modalidades de ensino, com base na conjugação de conhecimentos técnicos e tecnológicos com as suas práticas pedagógicas ${ }^{3}$. A inserção territorial, formação profissional, bem como as práticas científicas e tecnológicas são os principais aspectos definidores de sua existência, visando atingir o objetivo político de combate às desigualdades regionais ${ }^{3}$.

No âmbito dos IF é disponibilizada assistência à saúde dos discentes para viabilizar um ambiente favorável às habilidades e capacidades necessárias a formação pedagógica e cidadã. Todavia, não há formalização de uma política de saúde, sendo os setores de saúde balizados pela política de assistência estudantil característica dos IF. Portanto, não há um planejamento estratégico próprio de serviços de saúde em essência. Não obstante, os desafios dos serviços de saúde dos IF são 


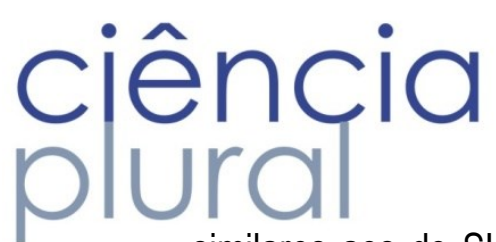

similares aos do SUS, visando transpor uma atenção apenas centrada no modelo biomédico para a vigilância da saúde atendendo ao panorama de tripla carga de doenças ${ }^{4}$.

A vigilância da saúde preconiza análise de situações de risco do território, destacando a heterogeneidade da população quanto às suas necessidades e acesso aos serviços de saúde 5 . Há uma concepção ampliada do processo saúde/doença/cuidado, onde saúde é tida como produto social. Portanto, essa proposição representa um deslocamento da tradicional base conceitual, limitada ao exclusivo controle e/ou erradicação dos agentes transmissores das doenças (biologicista), para uma ampliação que compreende as relações sociais como agentes relacionados às desigualdades em saúde 5 .

Mediante o comprometimento do IF com a transformação da realidade na perspectiva da igualdade e da justiça social, percebe-se a necessidade de uma política de saúde para orientar processos de trabalhos congruentes com esta missão. Logo, este estudo reporta a estruturação de uma política de saúde para a assistência estudantil do Instituto Federal Educação, Ciência e Tecnologia do Rio Grande do Norte (IFRN) numa concepção estratégica e participativa, a partir da construção de uma matriz de indicadores.

\section{Metodologia}

Trata-se de um relato acerca do processo de construção de uma política de saúde, caracterizando-se como uma pesquisa avaliativa em seu processo de desenvolvimento. Segundo Contandriopoulos et al. ${ }^{6}$ avaliar é julgar uma intervenção ou qualquer um dos seus componentes com o objetivo de auxiliar na tomada de decisões. Uma intervenção seria "um conjunto dos meios (físicos, humanos, financeiros, simbólicos) organizados em um contexto específico, em um dado momento, para produzir bens ou serviços com o objetivo de modificar uma situação problemática".

Diante da necessidade de elaborar o diagnóstico da realidade, objetivos pretendidos e a estratégia a ser adotada para a construção da política, este estudo, realizado no período de abril 2013 a Abril de 2014, foi conduzido conforme o percurso descrito a seguir.

Primeiramente, definiram-se as diretrizes da política, sendo necessário identificar a missão dos setores de saúde do IFRN, da Diretoria onde esses setores estão inseridos (Diretoria de Gestão de Atividades Estudantes - DIGAE) e da instituição para compreender como essas missões estariam relacionadas. Para isto, realizou-se análise documental do Plano de Assistência Estudantil ${ }^{7}$ e do Projeto Político-Pedagógico do IFRN8. Também foram consultados a Lei 11.892/20083, documentos relativos ao Programa Saúde na Escola ${ }^{9}$ e à série Planeja SUS10 para conhecer um programa de base 


\section{ciência plural}

nacional voltado à saúde estudantil e compreender o planejamento preconizado no SUS para adaptálos ao contexto dos IF.

O passo seguinte foi identificar as atribuições dos profissionais (médicos, técnicos de enfermagem, odontólogos), via análise dos editais de concursos e do Ofício Circular 015/2005/CGGP/SAA/SE/MEC, que dispõe sobre as atribuições de cargos no âmbito das IFES (Instituições Federais de Ensino Superior).

A seguir, iniciou-se a identificação dos elementos fundamentais à construção da política descritos por Fleury e Ouverney ${ }^{1}$. Para o diagnóstico situacional do público-alvo - os discentes dos IF -, consultaram-se levantamentos de saúde nacionais que envolvessem adolescentes na faixa etária preponderantemente englobada pelos IF (14 a 19 anos $)^{11,12}$, mediante ausência de levantamentos epidemiológicos realizados nos IF. Para tentar contextualizar a ocorrência das doenças e agravos presentes no universo dos adolescentes, foi realizada consulta ao Relatório Geral de caracterização socioeconômica dos alunos do IFRN ${ }^{13}$. Assim, as problemáticas-alvo para compor a agenda da Política de Saúde do IFRN foram: obesidade/alimentação/sedentarismo/ hipertensão/diabetes; sexualidade (DST/AIDS/gravidez/aborto); parasitoses/qualidade da água; álcool/drogas/tabagismo/violência; cárie/doença periodontal/perda dentária precoce; doenças imunopreveníveis; problemas oftalmológicos; doenças endêmicas e transtornos mentais.

Ainda, no afã do diagnóstico situacional, executou-se pesquisa para identificar os potenciais recursos do IFRN para efetivação da política de saúde. Assim, enviou-se questionário Google forms $₫$ para os coordenadores e servidores dos setores de saúde dos IF do RN $(n=85)$ investigando aspectos relacionados à infraestrutura (estrutura física do setor; equipamentos; instrumentais; materiais de consumo); gestão do trabalho (ações realizadas pelo setor; financiamento dessas ações; parcerias com as Secretarias municipais, estaduais e Ministério da Saúde; protocolos, normas e rotinas utilizados; estocagem de medicamentos; arquivamento de prontuários; realização de manutenção preventiva de equipamentos; presença de alvará sanitário para funcionamento do setor; de plano de gerenciamento de resíduos sólidos e da qualidade da água) e gestão de pessoas (quantitativo e funções dos profissionais; existência de ações de educação em saúde e de vigilância à saúde do trabalhador no setor). A partir deste conjunto de informações, delinearam-se diretrizes e objetivos da política de saúde em construção.

Em seguida, organizou-se matriz de indicadores para viabilizar uma avaliação acerca da implantação da Política. A matriz continha as problemáticas-alvo organizadas em três dimensões (atividades de educação em saúde, assistenciais e administrativas). Dentro dessas dimensões, havia 


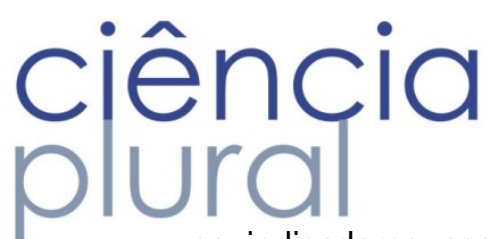

os indicadores associados aos objetivos traçados para a política. Para verificar a pertinência do conteúdo da matriz, optou-se por encaminhá-la via e-mail àqueles que a utilizariam no cotidiano dos processos de trabalho, tal qual preconizado na pesquisa avaliativa participativa pluralista ${ }^{14}$.

Assim, foi enviado aos 18 coordenadores da Assistência Estudantil uma carta convite contendo informações sobre a necessidade de construção de uma política de saúde para o IFRN e como esses coordenadores e profissionais de saúde poderiam contribuir para tal fim. Orientou-se que os coordenadores encaminhassem a matriz de indicadores aos profissionais do setor de saúde do seu Campus para que, individualmente, analisassem se os indicadores eram acessíveis (fáceis e rápidos de obter, de trabalhar e de compreender); confiáveis (apresentariam concordância entre aferições distintas realizadas em condições similares); oportunos (disponibilidade dos dados no local e tempo para quem deles necessita) e válidos (o dado ou informação mede o que se pretende medir). Foi solicitada, também, a sugestão de metas para cada um dos indicadores de acordo com a realidade vivenciada em cada Campus. E ainda, os coordenadores deveriam, posteriormente, indicar um representante de cada Campus para compor o grupo de discussão que validaria a configuração final da matriz proposta.

Posteriormente, em um encontro presencial, com o mínimo de um representante de cada Campus, foi realizada a pactuação dos indicadores e proposição de responsáveis para viabilização de cada indicador. Depois, iniciou-se processo de construção de instrumentos gerenciais que possibilitassem o monitoramento e avaliação das ações realizadas. Neste sentido, foram propostos a programação anual da saúde (PAS) e o relatório de execução das ações. Com o intuito de sintetizar o processo de construção da Política apresenta-se a figura 1. 


\section{ciência plural}

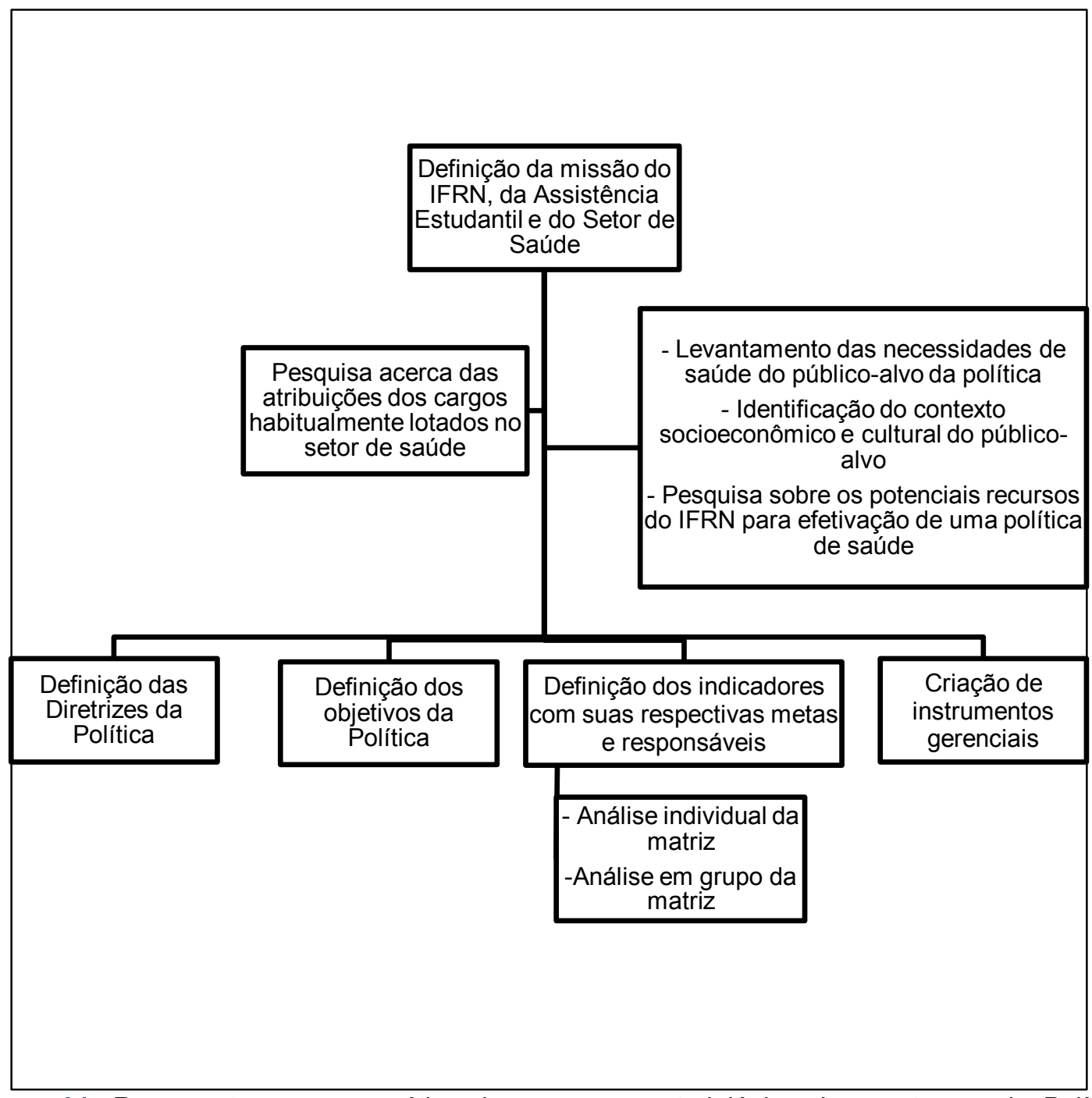

Figura 01: Representação esquemática do processo metodológico de construção da Política de Saúde Estudantil do IFRN. Natal-RN, 2018

\section{Resultados e Discussão}

\section{Público-alvo, Diretrizes e Objetivos}

Durante o encontro, supracitado, realizou-se a apresentação da missão do IFRN e da DIGAE, os quais constavam em documentos oficiais. Logo em seguida, foi apresentado um esboço da missão do setor de saúde do IFRN, a qual deveria estar congruente com as missões anteriores e contemplar em sua definição dois pontos-chave: o tipo de modelo de atenção e o público-alvo. Isto porque o enunciado de uma missão organizacional, segundo Cecílio ${ }^{15}$, deve ser norteado por três ideias basilares: o que é produzido, para quem é produzido e quais características do produto. 


\section{ciência \\ plural}

Quanto ao público-alvo, houve convergência de opiniões. De fato, a inclusão dos setores de saúde na DIGAE aponta os discentes como público-alvo. Todavia, servidores e terceirizados são assistidos em situações de urgência/emergência. Quanto aos produtos e suas características, foi debatido o modelo de atenção, havendo hegemonia absoluta quanto suplantar o modelo biomédico para o de vigilância da saúde (figura 2).

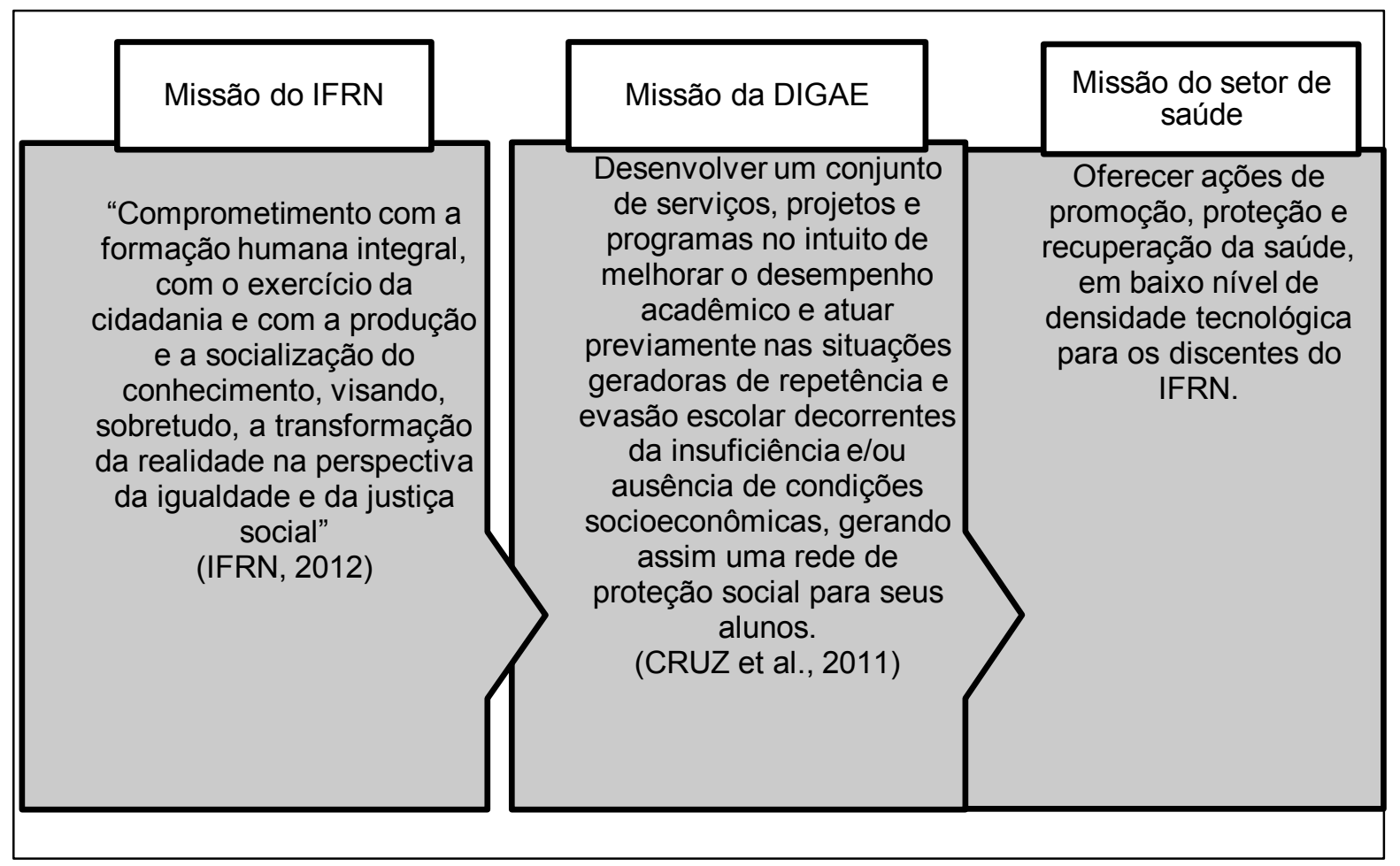

Figura 02: Identificação da missão do IFRN, da DIGAE e do setor de saúde segundo a análise documental realizada e discussão consensual dos servidores da saúde, diretores de atividades estudantis e gestão de pessoas e Reitor da instituição sob estudo. Natal-RN, 2018

Partiu-se debate das diretrizes e objetivos da Política. Quanto às diretrizes, não houve proposições para substituição ou readequações (quadro 1). 


\section{ciência plural}

Quadro 01: Identificação da missão do IFRN, da DIGAE e do setor de saúde segundo a análise documental realizada e discussão consensual dos servidores da saúde, diretores de atividades estudantis e gestão de pessoas e Reitor da instituição sob estudo. Natal-RN, 2018

\section{DIRETRIZ: PROMOVER A SAÚDE E A CULTURA DE PAZ, DE MANEIRA A COLABORAR COM 0 PROCESSO DE ENSINO-APRENDIZAGEM. \\ OBJETIVOS \\ -Diagnosticar precocemente, mediante o exame biomédico, situações de risco para sobrepeso/obesidade, diabetes e hipertensão. \\ -Acompanhar alunos acometidos por sobrepeso/obesidade, diabetes e/ou hipertensão em parceria com as equipes de saúde da família. \\ -Assegurar a oferta de alimentos saudáveis na merenda escolar. \\ -Assegurar o controle da qualidade da água na escola em parceria com a coordenação de manutenção. \\ -Desenvolver ações de proteção à saúde. \\ -Incentivar a prática esportiva.}

DIRETRIZ: FORTALECER O ENFRENTAMENTO DAS VULNERABILIDADES, NO CAMPO DA SAÚDE, QUE POSSAM COMPROMETER O PLENO DESENVOLVIMENTO ESCOLAR.

\section{OBJETIVOS}

-Desenvolver levantamentos epidemiológicos das condições de saúde do público-alvo.

-Firmar parcerias com as Secretarias Municipais e Estaduais de Saúde das localidades onde os Campi do IFRN se inserem para viabilizar o acesso dos discentes aos serviços de especialidades médicas e odontológicas não ofertadas nas escolas.

\section{DIRETRIZ: CONTRIBUIR PARA A CONSTRUÇÃO DE UM SISTEMA DE ATENÇÃO SOCIAL DENTRO}

DA ESCOLA, FOCANDO NA PROMOÇÃO DA CIDADANIA E DOS DIREITOS HUMANOS.

OBJETIVOS

- Incentivar a pesquisa no IFRN relacionada às questões de saúde.

DIRETRIZ: ARTICULAR EDUCAÇÃO E SAÚDE, AMPLIANDO O ALCANCE E O IMPACTO DAS AÇÕES, OTIMIZANDO A UTILIZAÇÃO DOS ESPAÇOS, EQUIPAMENTOS E RECURSOS DISPONÍVEIS.

\section{OBJETIVOS}

-Realizar ações de educação em saúde na perspectiva da educação popular e permanente.

DIRETRIZ: CONSTITUIR UMA POLÍTICA DE GESTÃO PARA OS SERVIÇOS DE SAÚDE DO IFRN. OBJETIVOS

-Avaliar os processos de trabalho desenvolvidos nos setores de saúde quanto à sua resolutividade.

-Discutir, a cada quatro anos, os objetivos da política de saúde.

-Desenvolver e instituir instrumentos de avaliação e monitoramento da política de saúde.

-Desenvolver tecnologias que propiciam a melhoria contínua dos processos de trabalho desenvolvidos nos setores de saúde.

-Normatizar o funcionamento dos serviços de saúde via protocolos, procedimentos operacionais padrão e manuais instrutivos

- Incentivar a participação da comunidade escolar (pais, alunos e professores) no planejamento das atividades do setor de saúde.

-Buscar a inserção de outras categorias de profissionais de saúde para melhor enfrentamento das vulnerabilidades.

De fato, as diretrizes são coerentes ao Plano de Assistência Estudantil do IFRN ${ }^{7}$ e a agenda das políticas públicas de saúde vigentes no Brasil, buscando redução das iniquidades em saúde, ênfase na promoção da saúde e instituição do planejamento estratégico. Portanto, os processos de trabalho desenvolvidos no âmbito do IFRN migrariam de uma proposição multiprofissional para uma interdisciplinar e intersetorial. 
Quanto aos objetivos da política, sintetizados no quadro 1, esses foram apresentados em relação direta aos indicadores propostos e às respectivas problemáticas onde os indicadores se inseriam na matriz. Após a discussão em grupo, um dentre os objetivos propostos foi excluído e outro foi modificado. No caso, o relacionado aos determinantes e condicionantes da saúde foi excluído dadas as colocações dos profissionais quanto à dificuldade, ineficácia e inviabilidade de tratarem desta temática. Foi argumentado que outros profissionais para além do setor de saúde, no caso àqueles do serviço social, estariam mais qualificados para trabalhar a questão dos determinantes e condicionantes. O objetivo modificado foi o relacionado à temática da dengue, posto que houve uma expansão da temática para doenças endêmicas em vista das peculiaridades dos territórios dos vários Campi do IFRN, houve a necessidade de contemplar doenças endêmicas diversas.

As temáticas selecionadas para compor os problemas-alvo da política estão relacionadas, preponderantemente, à educação popular em saúde com ênfase na educação sexual, educação alimentar, incentivo à prática esportiva, combate à utilização de drogas e redução da violência; vigilância da água; vigilância epidemiológica e promoção à saúde mental.

\section{Educação popular em saúde}

A Educação Popular em Saúde constitui-se como espaço de produção crítica de saberes e práticas onde os conhecimentos sobre Saúde Pública, em suas várias vertentes teóricas, são construídos no diálogo teórico e metodológico com outras áreas, buscando aportes na Educação, nas Ciências Sociais, na Psicologia, entre outras áreas de conhecimento que dizem respeito à Saúde Humana ${ }^{16}$.

Neste sentido, o conhecimento é construído no diálogo entre sujeitos e a aprendizagem é a construção desse conhecimento e a constatação da necessidade de transformação - a reconstrução da realidade. Assim, ao trabalhar-se a educação popular em saúde na escola, toma-se o espaço grupal como meio de construção da consciência coletiva mediada pelo diálogo entre equipe multiprofissional e educandos, tornando possível o encontro da reflexão com a ação, uma vez que o diálogo se impõe como caminho pelo qual os homens ganham significação enquanto sujeitos e conquistam o mundo para sua libertação mediante uma educação problematizadora e conscientizadora ${ }^{16}$.

A temática sexualidade vem sendo discutida no ambiente escolar desde a década de 1920, mas somente a partir da década de 1970 é que houve a inclusão nos currículos de $1^{\circ}$ e $2^{\circ}$ graus, com objetivo prioritário de formar o indivíduo na sua globalidade ${ }^{17}$. No entanto, foi a partir da década de 1980 que esta inclusão foi intensificada no Brasil mediante o aumento de casos de gravidez na 


\section{ciência plural}

adolescência e do risco de contaminação pelo HIV entre a população jovem ${ }^{17}$. Passadas duas décadas, o Departamento de Informática do SUS (DATASUS), em 2010, revela persistência de gravidez na adolescência, aborto, doenças sexualmente transmissíveis (DST), alta incidência de AIDS entre adolescentes e adultos jovens. Além disso, há o abuso e exploração sexual evidenciando a pertinência da temática da sexualidade em ambiente escolar.

Não obstante, foi orientado aos governos, na Conferência Internacional do Cairo em 1994, propiciar aos adolescentes informações e serviços adequados relativos à saúde sexual e reprodutiva. Assim, a discussão sobre sexualidade perpassa pela defesa dos direitos sexuais e reprodutivos e sugere uma atuação conjunta entre os serviços de saúde e o sistema educacional. A parceria ensinosaúde tornaria viável a criação de um projeto pedagógico sobre educação para a saúde sexual e reprodutiva de forma contínua e sistemática ${ }^{17}$.

Por sua vez, a educação alimentar é igualmente pertinente ao contexto da promoção da saúde em ambiente escolar, dada a sua potencialidade em desenvolver estratégias de estímulo a hábitos saudáveis, evitando agravos alvo das políticas públicas como a obesidade, diabetes e hipertensão arterial. O emprego da alimentação saudável deve compreender o uso de alimentos variados, seguros, que respeitem a cultura, as tradições e os hábitos alimentares saudáveis, contribuindo para 0 crescimento e o desenvolvimento dos alunos e para a melhoria do rendimento escolar, em conformidade com sua faixa etária e seu estado de saúde, inclusive dos que necessitam de atenção específica ${ }^{18}$. Muitas das características ou dos hábitos referentes ao estilo de vida do adulto são adquiridos e/ou consolidados na infância e na adolescência. Daí a importância de ações educativas para evitar futuros problemas de saúde.

Neste sentido, a criação de políticas voltadas ao esclarecimento da população quanto ao consumo de alimentos e bebidas e incentivadoras da atividade física é essencial. Neste sentido, a escola é uma importante aliada para a concretização de ações de promoção de saúde para os adolescentes, uma vez que concentra grande parte dos indivíduos nesta fase da vida.

\section{Vigilância epidemiológica}

Segundo Garbin et al. ${ }^{19}$, é na adolescência que o indivíduo experimenta os melhores índices de saúde e vitalidade, que podem ser mantidos e/ou melhorados, dependendo da percepção e valorização que os mesmos adquirem sobre 0 assunto. 0 adolescente, ao mesmo tempo em que experimenta nesta fase mudanças biológicas, cognitivas, emocionais e sociais e vivencia um importante momento para a adoção de novas práticas, comportamentos e ganho de autonomia, também está exposto a 


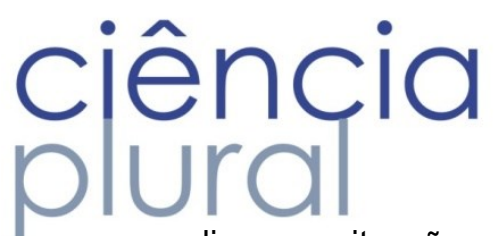

diversas situações que envolvem riscos para a saúde, tais quais: tabagismo, consumo de álcool e outras drogas, alimentação inadequada, sedentarismo e atividade sexual precoce, que na maioria das vezes ocorre de forma desprotegida. Frente a essa realidade, são observados indicadores crescentes de agravos à saúde do adolescente, como a maior incidência de HIVIAIDS, gravidez de alto risco, problemas nutricionais, violência, uso e abuso de bebidas alcoólicas e substâncias psicoativas ${ }^{20}$. Além disso, alguns destes fatores estão associados ao desenvolvimento da maioria das doenças crônicas não transmissíveis como as cardiovasculares, diabetes e câncer, que lideram as causas de óbito na vida adulta no país ${ }^{21}$.

Em adição, o SB Brasil 2010 revela que os adolescentes (15 a 19 anos) apresentam em média 4,25 dentes com experiência de cárie dentária; 49,1\% desses apresentam pelo menos um sextante com doença periodontal, dos quais a presença de cálculo foi a alteração periodontal mais presente $(28,4 \%)$. Além disso, as prevalências de oclusopatias severa e muito severa, nesta faixa etária, têm sido elevadas, 6,6\% e 10,3\%, respectivamente ${ }^{11}$. Embora a maioria das doenças bucais não seja fatal, elas impactando negativamente no desempenho das atividades diárias e na qualidade de vida dos indivíduos por causarem dor, sofrimento, desconforto psicológico e privações sociais, acarretando prejuízos em nível individual e coletivo22.

Por reconhecer os potenciais impactos na qualidade de vida que os agravos à saúde e seus fatores de risco podem ocasionar na adolescência e na futura vida adulta, a minimização ou prevenção dos mesmos se apresentam como importantes medidas de saúde pública. Para tanto, é necessário um conjunto de ações que proporcionem o conhecimento e a detecção precoce deste conjunto de fatores de risco e agravos da saúde individual e/ou coletiva, com a finalidade de recomendar a adoção de medidas de prevenção e controle, conforme o conceito de vigilância epidemiológica ${ }^{23}$. Logo, na política de saúde desenvolvida no IFRN, buscou-se a vigilância epidemiológica numa abordagem estratégica de atividade de inteligência ${ }^{23}$ para observação contínua da distribuição e das tendências da incidência de doenças na adolescência mediante a coleta sistemática (através de um sistema de informação próprio da instituição), a consolidação e a avaliação de dados de morbidade, assim como de outros dados relevantes para a saúde.

A vigilância epidemiológica é tomada, nesta política, como ferramenta importante para priorização das ações em saúde, no sentido de produzir informações que sirvam como subsídios para estimar a magnitude dos problemas de saúde e aplicar oportunamente a prevenção apropriada 23 . Assim, constitui-se em um instrumento fundamental para o planejamento, organização e avaliação das intervenções no âmbito dos serviços de saúde do IFRN. 


\section{ciência plural}

Outro ponto essencial na política, associado à vigilância epidemiológica, diz respeito à imunização. A importância da vacina, imunização ativa, na proteção à saúde e prevenção de doenças imunopreveníveis, é reconhecida mundialmente. Como consequência, as autoridades de saúde, em todo o mundo, assim como no Brasil, estabeleceram calendários específicos de vacinas de acordo com a faixa etária. Apesar disto, o público adolescente tem sido negligenciado quanto à vacinação. Uma parcela considerável de adolescentes e adultos jovens não tem imunidade para certas doenças endêmicas. A maioria dos adolescentes não toma os reforços de algumas vacinas, recomendados nos casos em que a imunização perde o efeito com o tempo. Em outras situações, a vacina pode não ter sido aplicada durante a infância pela inexistência da dose na época. Portanto, numa política de saúde que contemple a saúde integral de adolescentes, é fundamental abordar a imunização nesta faixa etária.

\section{Vigilância ambiental em saúde}

A incorporação da vigilância ambiental no campo das políticas públicas de saúde é uma demanda relativamente recente no Brasil. A influência que o ambiente possui na saúde das populações deve ser assunto das atuais políticas de saúde pautadas no modelo de vigilância à saúde. Cabe à vigilância ambiental em saúde investigar o conjunto de fatores ambientais que atuam sobre a população e as relações sociais que estruturam estes fatores ${ }^{24}$.

Historicamente, as ações de saneamento têm concentrado maior interesse do setor de saúde entre as demais intervenções cunho ambiental porque a água, apesar de ser um elemento essencial à vida, pode oferecer riscos à saúde em face de sua má qualidade e quantidade. Ela tem influência direta sobre a saúde, a qualidade de vida e o desenvolvimento do ser humano, exigindo atenção quanto aos fatores que podem interferir negativamente em sua qualidade. Neste sentido, destaca-se a importante da inclusão da temática vigilância à qualidade da água nesta política de saúde.

\section{Promoção à saúde mental}

A temática da saúde mental merece particular atenção na faixa etária abrangida pelos institutos, pois a transição da infância para a adolescência parece ser ponto de partida para o desenvolvimento de problemas psicológicos. A partir desta fase, são vivenciados conflitos internos que podem comprometer diretamente a saúde mental25.

Durante a última década, estudos em diversos países têm mostrado o aumento na proporção de adolescentes com problemas psicossomáticos ${ }^{25}$. Problemas de saúde mental em adolescentes que 


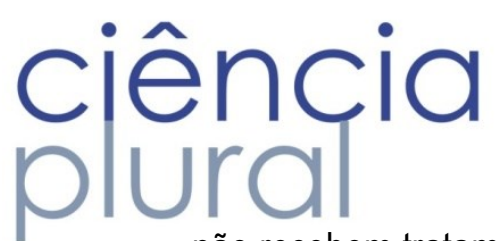

não recebem tratamento estão associados a baixos níveis de realização educacional, desemprego, uso de drogas, comportamentos de risco, criminalidade, saúde sexual e reprodutiva precárias, automutilação e cuidados pessoais inadequados. Por outro lado, adolescentes cujas necessidades em saúde mental são reconhecidas têm melhor adaptação em termos sociais, melhor desempenho na escola e são mais propensos a se tornarem adultos mais adaptados e produtivos que aqueles cujas necessidades não são atendidas ${ }^{25}$.

A ocorrência da maioria dos problemas de saúde mental é determinada por um conjunto de fatores e o conhecimento desses fatores, tanto os de risco como os de proteção, permite o desenvolvimento de estratégias e ações de prevenção e tratamento dos problemas de saúde mental.

Acredita-se que a escola pode funcionar como fator de risco ou de proteção ao desenvolvimento de problemas na saúde mental dos estudantes. Pinto et al. ${ }^{25}$ encontraram vários fatores de risco relacionados à escola, como: baixo desempenho ou abandono escolar, bullying, vivência de discriminação e racismo, e pressão social dos pares e da vida acadêmica. Por outro lado, o ambiente escolar pode desempenhar importante papel como território propício a ajudar os adolescentes a aumentar suas habilidades sociais, ampliar a capacidade de solucionar problemas e intensificar a autoconfiança, o que pode amenizar os problemas em saúde mental e evitar comportamentos violentos e de risco. Portanto, a saúde mental, enquanto temática incorporada à política de saúde do IFRN, tem como foco à promoção da saúde e prevenção de doenças e agravos.

Lidar com problemas de saúde mental no ambiente escolar não é uma tarefa fácil, mas tampouco torna imprescindível a presença de especialistas em saúde mental. A realização de ações educativas, reflexivas e de abordagem psicossocial ajuda na identificação precoce dos hábitos inadequados e de alterações corporais e psíquicas através da investigação da saúde mental, podendo promover melhor qualidade de vida. Por isso, as ações de prevenção de agravos à saúde do adolescente, estimuladas por esta política de saúde, pretendem romper com as visões biomédica e tecnicista, que não consideram a integralidade do adolescente como ser em formação. É preciso considerar a transdisciplinaridade do tema, uma vez que os problemas psíquicos e emocionais que afetam 0 adolescente podem desencadear outras implicações como gravidez indesejada, uso de drogas, envolvimento em situações de violência, etc, além de repercutir nas fases seguintes da sua vida 25 .

Portanto, a política de saúde estudantil do IFRN foi pautada no conceito de que a escola é um espaço de grande importância para promoção e prevenção de agravos, através de dinâmicas participativas, possibilitando a discussão e reflexão sobre as mais diversas temáticas que afetam a vida dos seus discentes. 


\section{ciência plural}

\section{Indicadores}

Os indicadores foram discutidos um a um e, em seguida, pactuadas suas respectivas metas. No geral, 07 indicadores foram modificados quanto à escrita, 10 modificados quanto à forma de medição, 09 foram excluídos e 03 foram criados.

Partindo-se para uma análise dos indicadores, dentre os de educação em saúde, aqueles relacionados a "incentivar o cuidado com a saúde" e "realizar atividades sistemáticas de promoção, prevenção e recuperação da saúde" foram excluídos, pois se considerou que eles englobavam todos os demais indicadores de educação em saúde e não direcionavam as condições de saúde/temáticas consideradas prioritárias a serem abordadas com o público-alvo da escola. Dois indicadores relacionados à imunização foram fusionados em um único (informar os alunos sobre a importância das imunizações e colaborar com campanhas vacinais de vinculação nacional) (quadros 2 e 3 ).

Quanto aos indicadores assistenciais, três foram removidos da matriz. 0 indicador acerca da saúde auditiva foi removido devido a inviabilidade de captar o dado necessário para sua construção, posto que o setor de saúde não dispõe de equipamentos para detecção precoce de alterações auditivas e sua aquisição seria de alto custo frente ao benefício alcançado. Já o indicador de assistência médica às gestantes (Dar suporte e orientação às gestantes) foi excluído pela existência do programa de pré-natal do SUS que viabilizaria a informação pretendida. Por fim, o indicador de "diagnóstico precoce de transtornos mentais" foi retirado devido a necessidade de extensas capacitações para que os profissionais estivessem habilitados a realizar tal diagnóstico. Ainda na categoria dos indicadores assistenciais, aqueles que abordavam a temática de gestão em saúde (Existência de termo de parceria firmado com as secretarias municipais e estadual para encaminhamentos dos alunos para as especialidades médicas e odontológicas e Número de novos códigos de vagas de profissionais da saúde adquiridos por campus no período de 04 anos) foram realocados para a categoria atividades administrativas (Quadros 2 e 3 ).

$\mathrm{Na}$ categoria dos indicadores relacionados às atividades administrativas, aquele ligado ao cadastro dos setores de saúde ao Cadastro Nacional de Estabelecimentos de Saúde (CNES) do Ministério da Saúde foi excluído em virtude das dúvidas acerca dos potenciais benefícios e ônus deste registro para os setores de saúde do instituto. Diante da falta de clareza quanto aos deveres impostos aos setores com esse cadastro e da dubiedade dessas questões, o grupo deliberou pela exclusão do indicador. Nesta categoria, houve a inclusão de indicadores relacionados à pesquisa científica (Número 


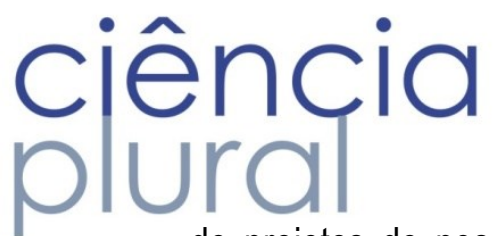

de projetos de pesquisa submetidos aos editais de instituições de pesquisa) e à normatização dos setores (Existência e distribuição de manual de boas práticas para todos os setores de saúde dos campi). Foram incluídos, ainda, aqueles realocados da categoria previamente discutida.

Ao final da discussão, a matriz anteriormente composta por 42 indicadores distribuídos nas categorias de atividades de educação em saúde $(n=18)$, assistenciais $(n=07)$ e administrativas $(n=17)$ passou a ser composta por 37 indicadores - educação em saúde: 10; atividades assistenciais: 07; atividades administrativas: 20 - selecionados para avaliar a implantação e execução da política de saúde proposta.

Quadro 2 - Indicadores selecionados para avaliar a implantação e execução da Política de Saúde Estudantil distribuídos segundo as temáticas relacionadas e as atividades de educação em saúde, atividades assistenciais e atividades administrativas.

\begin{tabular}{|c|c|c|c|}
\hline Temáticas & Educação em saúde & $\begin{array}{l}\text { Atividades } \\
\text { assistenciais }\end{array}$ & $\begin{array}{c}\text { Atividades } \\
\text { administrativas }\end{array}$ \\
\hline $\begin{array}{l}\text { Obesidade/alimentação/s } \\
\text { edentarismo/hipertensão/ } \\
\text { diabetes }\end{array}$ & $\begin{array}{l}\text { - Percentual de alunos do } \\
\text { campus atingidos por ações } \\
\text { que abordem o tema da } \\
\text { reeducação alimentar durante } \\
01 \text { ano. } \\
\text { - Percentual de alunos do } \\
\text { campus atingidos por ações } \\
\text { que abordem o tema da } \\
\text { hipertensão durante } 01 \text { ano. } \\
\text { - No de campanhas } \\
\text { relacionadas à prática } \\
\text { esportiva durante } 01 \text { ano. }\end{array}$ & $\begin{array}{l}\text { - Percentual de alunos } \\
\text { ingressantes no ensino } \\
\text { médio integrado } \\
\text { submetidos a exame } \\
\text { biomédico durante } 01 \\
\text { ano. } \\
\text { - Percentual de alunos } \\
\text { diagnosticados com } \\
\text { diabetes/obesidade/sobr } \\
\text { epeso/hipertensão } \\
\text { assistidos no setor de } \\
\text { saúde. }\end{array}$ & $\begin{array}{lr}\text { - Percentual de } \\
\text { itens relacionados a } \\
\text { alimentos } & \\
\text { saudáveis } & \\
\text { adquiridos no } \\
\text { processo licitatório } \\
\text { e na agricultura } \\
\text { familiar } & \\
& \\
\text { - Padronização do } \\
\text { contrato } & \text { da } \\
\text { refeição/cantina }\end{array}$ \\
\hline Sexualidade & $\begin{array}{l}\text { - No de ações realizadas com } \\
\text { o tema de saúde reprodutiva } \\
\text { durante } 01 \text { ano. } \\
\text { - Existência de um grupo de } \\
\text { discussão para trabalhar a } \\
\text { temática da sexualidade. }\end{array}$ & $\begin{array}{l}\quad \text { Percentual de } \\
\text { gestantes assistidas pela } \\
\text { odontologia (tratamento } \\
\text { odontológico concluído) } \\
\text { durante o ano. }\end{array}$ & \\
\hline $\begin{array}{l}\text { Parasitoses/qualidade da } \\
\text { água }\end{array}$ & & & $\begin{array}{lr}\text { - Número de laudos } \\
\text { de análise } \\
\text { microbiológica } \\
\text { físico-química } \\
\text { durante } 01 \text { ano. } \\
\\
\quad \quad \text { Número de } \\
\text { higienizações do } \\
\text { reservatório de } \\
\text { água, com registro. }\end{array}$ \\
\hline
\end{tabular}




\begin{tabular}{|c|c|c|c|}
\hline $\begin{array}{l}\text { Álcool/drogas/tabagismo/ } \\
\text { violência }\end{array}$ & $\begin{array}{l}\text { - Número de reuniões } \\
\text { temáticas }\end{array}$ & & \\
\hline $\begin{array}{l}\text { Cárie/doença } \\
\text { periodontal/perda } \\
\text { dentária precoce }\end{array}$ & & $\begin{array}{l}\text { - Proporção de alunos } \\
\text { com tratamento } \\
\text { concluído em relação ao } \\
\text { total de alunos atendidos } \\
\text { durante } 01 \text { ano. } \\
\text { - Percentual dos alunos } \\
\text { ingressantes no ensino } \\
\text { médio integrado } \\
\text { submetidos à exame } \\
\text { bucal (índice CPO-D) } \\
\text { durante a exame } \\
\text { biomédico no período de } \\
01 \text { ano. }\end{array}$ & \\
\hline $\begin{array}{l}\text { Doenças } \\
\text { imunopreveníveis }\end{array}$ & $\begin{array}{l}\text { - Percentual dos cartões de } \\
\text { vacina dos alunos } \\
\text { ingressantes no ensino médio } \\
\text { integrado analisados pela } \\
\text { equipe de enfermagem do } \\
\text { campus no período de } 01 \text { ano. }\end{array}$ & $\begin{array}{l}\text { - Percentual de discentes } \\
\text { imunizados do ensino } \\
\text { médio integrado durante } \\
01 \text { ano. }\end{array}$ & \\
\hline $\begin{array}{l}\text { Problemas } \\
\text { oftalmológicos }\end{array}$ & & $\begin{array}{l}\text { - Percentual de alunos } \\
\text { ingressantes no ensino } \\
\text { médio integrado } \\
\text { submetidos a exame } \\
\text { biomédico durante } 01 \\
\text { ano. }\end{array}$ & \\
\hline $\begin{array}{l}\text { Doenças endêmicas } \\
\text { Transtornos mentais }\end{array}$ & $\begin{array}{l}\text { - Número de ações educativas } \\
\text { voltadas para as questões } \\
\text { relacionadas às doenças } \\
\text { endêmicas características da } \\
\text { região dos campi no período } \\
\text { de } 1 \text { ano. } \\
\text { - Número de reuniões sobre a } \\
\text { temática de transtornos } \\
\text { mentais com professores e } \\
\text { discentes no período de } 01 \\
\text { ano. }\end{array}$ & & \\
\hline
\end{tabular}


Quadro 3 - Indicadores relacionados à gestão em saúde do IFRN selecionados para avaliar a implantação e execução da Política de Saúde Estudantil distribuídos segundo as temáticas relacionadas e as atividades de educação em saúde e atividades administrativas.

\begin{tabular}{|c|c|c|}
\hline Temáticas & Educação em saúde & Atividades administrativas \\
\hline Planejamento & & $\begin{array}{l}\text { - Existência de Política de Saúde Plurianual } \\
\text { (PSP) } \\
\text { - Existência da Programação Anual de Saúde } \\
\text { (PAS) }\end{array}$ \\
\hline Financiamento & & $\begin{array}{l}\text { - Existência de ferramenta do SUAP } \\
\text { disponibilizada com esta função de } \\
\text { comunicação entre os profissionais de saúde } \\
\text { para troca e doação de materiais de consumo e } \\
\text { permanentes. } \\
\text { - Percentual de profissionais que utilizam a } \\
\text { ferramenta disponibilizada no SUAP para troca } \\
\text { de informações acerca da aquisição de } \\
\text { materiais, equipamentos e contrato entre os } \\
\text { campi. } \\
\text { - Existência de ferramenta do SUAP } \\
\text { disponibilizada com esta função de visualização } \\
\text { de catálogo de materiais e equipamentos } \\
\text { médicos-odontológicos para compra anual. }\end{array}$ \\
\hline Participação social & $\begin{array}{l}\text { - Existência de um caixa de } \\
\text { sugestão no setor de saúde } \\
\text { para verificar a percepção do } \\
\text { discente sobre o atendimento. }\end{array}$ & $\begin{array}{l}\text { - Existência de termo de parceria com as } \\
\text { Secretarias de Saúde municipais elou } \\
\text { estaduais, assinado e em vigência, para } \\
\text { encaminhamento dos alunos às especialidades } \\
\text { médicas e odontológicas. }\end{array}$ \\
\hline Gestão do trabalho & $\begin{array}{l}\text { - } \mathrm{N}^{0} \text { de ações de educação } \\
\text { permanente para os } \\
\text { profissionais de saúde } \\
\text { realizadas no período de } 12 \\
\text { meses. }\end{array}$ & $\begin{array}{l}\text { - Número de novos códigos de vaga de } \\
\text { profissionais da saúde adquiridos por campus } \\
\text { no período de } 4 \text { anos. }\end{array}$ \\
\hline Informação em saúde & & $\begin{array}{l}\text { - Existência de Módulo Saúde no SUAP } \\
\text { (prontuário eletrônico). } \\
\text { - Percentual de profissionais do setor de saúde } \\
\text { que utilizam o sistema de informação no módulo } \\
\text { SUAP. } \\
\text { - Existência de Grupo de pesquisa da saúde } \\
\text { cadastrado na CAPES. } \\
\text { - No de projetos de pesquisa submetidos aos } \\
\text { editais de instituições de pesquisas. }\end{array}$ \\
\hline Infraestrutura & & $\begin{array}{l}\text { - Existência de Alvará sanitário em vigência. } \\
\text { - Existência de Projeto Básico de Arquitetura } \\
\text { aprovado pela vigilância sanitária. } \\
\text { - Existência e Distribuição de Manual de Boas } \\
\text { Práticas para todos os setores de saúde dos } \\
\text { campi do RN. } \\
\text { - Existência e Implementação de Plano de } \\
\text { Gerenciamento de Resíduos. } \\
\text { - Existência de norma técnica de funcionamento } \\
\text { dos setores de saúde. }\end{array}$ \\
\hline
\end{tabular}




\section{Instrumentos Gerenciais}

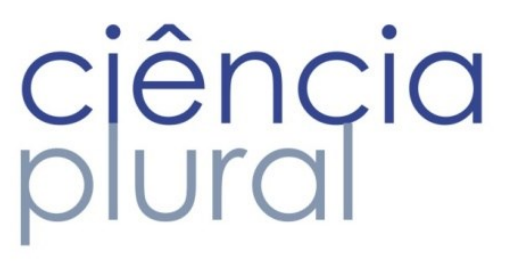

Em linhas gerais, a PAS é estruturada em dois tópicos: caracterização do campus e ações programadas. Na caracterização, devem ser descritos os cursos oferecidos e a quantidade de discentes inseridos em cada um deles, especificar os municípios onde ess discentes residem e a composição quali e quantitativa da equipe de saúde do setor de saúde do campus. Nas ações programadas, deve ser especificado aquilo que o Campus pretende desenvolver em 12 meses, relacionando essas ações a um objetivo (pertinente aos englobados pela política de saúde), um indicador, meta, previsão temporal de realização das atividades, especificação das parcerias requeridas quando necessário e dos recursos necessários para o desenvolvimento das ações.

Já o Relatório de Execução estrutura-se em três tópicos: ações executadas, ações não executadas e considerações finais. No primeiro tópico deve ser especificada cada ação desenvolvida, indicando o que foi feito, o público atingindo, os recursos utilizados e os possíveis impactos. No segundo, deve ser indicada cada ação programada na PAS mas não realizada, explicitando a justificativa da não realização. No último tópico, devem ser descritas as recomendações como revisão de indicadores, proposição de novas metas, reprogramação, proposição de novos objetivos tidos como necessários para atingir o previsto na PAS.

Em síntese, a Política de Saúde proposta apresenta como principal desafio sua legitimidade para o IFRN como um todo, mas, sobretudo, para aqueles diretamente envolvidos com sua concretização os profissionais de saúde. No intuito de viabilizar esta legitimidade, visitas foram realizadas em cada Campus do IFRN para apresentar a matriz final de indicadores, discutir estratégias para alcance das metas, orientar a utilização dos instrumentos de gestão propostos (PAS e relatório de execução) e discutir a governança de cada Campus para sua execução. Essas visitas foram agendadas com as diretorias locais para que houvesse a sua participação, bem como dos profissionais de saúde, coordenador da assistência estudantil e representantes da equipe pedagógica. De modo geral, houve uma aceitação maciça da Política e proposição de estratégias importantes para sua efetivação. Essas estratégias são relativas à participação social, já que a política fundamenta-se na construção participativa. Foi pactuada a realização de fóruns de discussão semestrais via videoconferência com os grupos de servidores envolvidos com as ações programadas a fim de compartilhar experiências e novas deliberações. Ainda na perspectiva de participação social, foi reforçada a necessidade de criar um canal de comunicação entre equipe de saúde, professores e discentes para trabalhar temáticas relacionadas à saúde, o que facilitaria o reconhecimento do papel social do IFRN e a adesão da comunidade nas ações programadas. 
O gerenciamento da política de saúde será realizado de forma descentralizada, posto que cada Campus na pessoa do coordenador de atividades estudantis fará sua avaliação processual. Contudo, foi discutida a necessidade de avaliar em conjunto os resultados alcançados por cada Campus. Esta avaliação em conjunto será realizada por um Conselho gestor composto especificamente com esta função cujos participantes serão eleitos pelos Campi. No primeiro ano de avaliação da política, o Conselho será composto pelos idealizadores da política. Assim, percebe-se a veemência do reconhecimento da política pelo IFRN, posto que sua implantação implicará mudanças organizacionais e, por conseguinte, apoio institucional para efetivá-las.

\section{Considerações Finais}

A política de saúde proposta pressupõe mudanças organizacionais não só nos setores de saúde, mas no IFRN, uma vez que as modificações dos processos de trabalho nos setores de saúde perpassam por questões individuais, mas, sobretudo, da instituição. De fato, a viabilização das estratégias propostas requer a institucionalização da educação em saúde, da pesquisa científica, dos processos de avaliação/planejamento/monitoramento, normatização do funcionamento dos serviços e o desenvolvimento de ações intersetoriais. Espera-se que a Política proposta contribua para o melhor desempenho acadêmico dos alunos e permanência dos mesmos na instituição, mediante oferta de ações promotoras, protetoras e de recuperação da saúde, utilizando tecnologias de baixa densidade tecnológica e essencialmente relacionais. Assim, pretende-se instituir um modelo balizado no cuidado à saúde que, potencialmente, contribua para formação de um sistema de proteção social ao aluno. 


\section{Referências}

1. Fleury S, Ouverney AM. Política de saúde: uma política social. In: Giovanella L, Escorel S, Lobato LVC, Noronha JC, Carvalho Al (org.). Políticas e sistema de saúde no Brasil. Rio de Janeiro: Fiocruz; 2008. p.23-64.

2. Santos SSBS, Melo CMMM, Costa HOG, Tanaka OU, Ramos FM, Santana MCC, Trindade BG. Avaliação da capacidade de gestão descentralizada da vigilância epidemiológica no estado da Bahia. Ciên Saúde Colet. 2012; 17(4):873-882.

3. Brasil. Lei $n^{0} 11.892$, de 30 de dezembro de 2008a. Institui a Rede Federal de Educação Profissional, Científica e Tecnológica, cria os Institutos Federais de Educação, Ciência e Tecnologia, e dá outras providências. Diário Oficial da União 2008; 30 dez.

4. Mendes EV. As redes de atenção à saúde. Ciên Saúde Colet. 2010; 15(5):2297-2305.

5. Santiago AC, Fracolli LA, Zoboli ELCP, Silva RMV. Indicadores sociais e de saúde para a operacionalização da Vigilância à Saúde. Rev Esc Enferm USP 2008; 42(4):798-803.

6. Contandriopoulos A, Champagne F, Denis J, Pineault R. Avaliação na área de saúde: conceitos e métodos. In: Hartz Z. Avaliação em saúde: dos modelos conceituais à prática na análise da implantação de programas. Rio de Janeiro: FIOCRUZ; 1997. p.29-48.

7. Instituto Federal de Educação, Ciência e Tecnologia do Rio Grande do Norte-IFRN. Plano de Assistência Estudantil. Natal; 2010.

8. Instituto Federal de Educação, Ciência e Tecnologia do Rio Grande do Norte-IFRN.. Projeto Político-Pedagógico do IFRN: uma construção coletiva. Natal; 2012.

9. Brasil. Manual instrutivo - Programa saúde na escola 2013. Brasília: Ministério da Educação e da Saúde; 2013a.

10. Brasil. Sistema de planejamento do SUS: uma construção coletiva- instrumentos básicos. Brasília: Ministério da Saúde; 2009.

11. Brasil. Pesquisa Nacional de Saúde Bucal SB Brasil 2010: Resultados principais. Brasília: Ministério da Saúde; 2011. 92p.

12. Instituto Brasileiro de Geografia e Estatística-IBGE. Sinopse do Censo Demográfico 2010. [acessado agosto de 2014]. Disponível em: http://portal.mte.gov.br/data/files/8A7C816A2E7311D1013003524D7B79E4/IBGE_CENSO2010_sinops e.pdf

13. Instituto Federal de Educação, Ciência e Tecnologia do Rio Grande do Norte-IFRN. Relatório Geral de caracterização socioeconômica dos alunos do IFRN 2013. [acessado setembro de 2013]. Disponível em: http://www.suap.ifrn.edu.br 


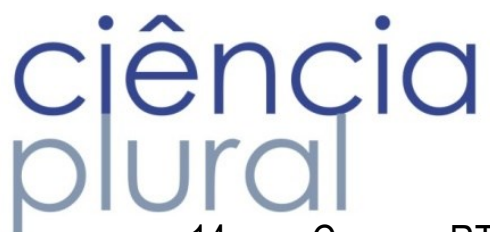

14. Campos RTO, Miranda L, Gama CAP, Ferrer AL, Diaz AR, Gonçalves L, Trapé TL. Oficinas de construção de indicadores e dispositivos de avaliação: uma nova técnica de consenso. REVIPSI 2010; 10(1):221-241.

15. Cecílio LCO. Uma sistematização e discussão de tecnologias leves de planejamento estratégico aplicada ao setor governamental. In: Merhy EE, Onocko R (org.). Agir em Saúde: um desafio para o público. São Paulo: HUCITEC; 1997. p.151-167.

16. Oliveira MW. Educação Popular e Saúde. Rev. Ed. Popular 2007; 6(1):73-83.

17. Brasil. Caderno de atenção básica 26: Saúde Sexual e saúde reprodutiva. Brasilia: Ministério da Saúde; 2013b.

18. Silva CAM, Marques LA, Bonomo E, Bezerra OMPA, Corrêa MS, Passos LSF, Souza AA, Barros BF, Souza DMS, Reis JA, Andrade NG. O Programa Nacional de Alimentação Escolar sob a ótica dos alunos da rede estadual de ensino de Minas Gerais, Brasil. Ciên saúde colet. 2013; 18(4):963-969.

19. Garbin CAS, Garbin AJ, Moimaz SAS, Gonçalves PE. A saúde na percepção do adolescente. Physis 2009; 19(1): 227-238.

20. Medeiros SB, Lima KYN, Moreira PNO, Tourinho FSV, Santos VEP. Saúde do adolescente escolar: uma pesquisa documental. J. Res. Fundam. Care 2013; 6(3):1105-1116.

21. Brasil. Pesquisa Nacional de Saúde do Escolar 2012. Rio de Janeiro: Ministério da Saúde, $2013 c$.

22. Silveira MF, Marôco JP, Freire RS, Martins AMEBL, Marcopito LF. Impacto da saúde bucal nas dimensões física e psicossocial: uma análise através da modelagem com equações estruturais. Cad. Saúde Pública 2014; 30(6):1-15.

23. Rodrigues-Júnior AL. A inteligência epidemiológica como modelo de organização em saúde. Ciên saúde colet 2012; 17(3):797-805.

24. Barcellos C, Quitério LAD. Vigilância ambiental em saúde e sua implantação no Sistema Único de Saúde. Rev Saude Pública 2006; 40(1): 170-177.

25. Pinto ACS, Luna IT, Silva AA, Pinheiro PNC, Braga VAB, Souza AMA. Fatores de risco associados a problemas de saúde mental em adolescentes: revisão integrativa. Rev Esc Enferm. 2014; 48(3):555-64.

Submetido: $10 / 08 / 2018$

Aprovado: 30/12/2018 